\title{
Identificação de Danos Estruturais a partir de um Modelo de Superfície de Resposta ${ }^{\dagger}$
}

\author{
I.C.S.S. RANGEL ${ }^{*}$, L.S. RANGEL e L.T. STUTZ
}

Recebido em 31 agosto, 2014 / Aceito em 9 setembro, 2016

\begin{abstract}
RESUMO. Neste trabalho, aborda-se o problema de identificação de danos estruturais a partir das frequências naturais. Na formulação do problema de identificação de danos é utilizado o Modelo de Superfície de Reposta (MSR) em substituição a um Modelo de Elementos Finitos (MEF) da estrutura. No presente trabalho, a estrutura simulada trata-se de uma viga de Euler-Bernoulli simplesmente apoiada, no qual o problema inverso em questão é definido como um problema de minimização, onde objetivo é minimizar uma função de erro definida a partir dos dados experimentais e de resultados analíticos previstos pelo MSR. A utilização do método Evolução Diferencial (ED) no problema de identificação de danos é considerado. Frente aos resultados numéricos obtidos, a estratégia adotada mostrou-se capaz de localizar e quantificar os danos com elevada acurácia.
\end{abstract}

Palavras-chave: identificação de danos estruturais, frequências naturais, modelo de superfície de resposta.

\section{INTRODUÇÃ̃o}

A identificação de danos estruturais é uma questão de fundamental importância na engenharia, visto que uma estrutura está sujeita a processos de deterioração e a ocorrência de danos durante a sua vida útil. A presença de danos compromete o desempenho e a integridade estrutural, podendo colocar vidas humanas em risco e resultam em perdas econômicas consideráveis.

O contínuo monitoramento da estrutura e a identificação de danos num estágio inicial contribuem para a redução dos custos de manutenção e de reparo, além de aumentar sua confiabilidade e sua vida útil. Neste caso, análises numéricas e experimentais podem ser realizadas com intuito de fornecer recursos para uma correta avaliação da integridade da estrutura, podendo assim, estabelecer critérios da utilização da estrutura com segurança.

Os métodos de identificação de danos geralmente são baseados em: dados modais (análise modal), dados no domínio do tempo e dados no domínio da frequência. Os métodos de identificação

$†$ Trabalho apresentado no XXXV Congresso Nacional de Matemática Aplicada e Computacional.

*Autor correspondente: Isabela Cristina da Silveira e Silva Rangel.

Instituto Politécnico, IPRJ, UERJ - Universidade do Estado do Rio de Janeiro, 28610-974, Nova Friburgo, RJ, Brasil.

E-mails: isabelacssilva@yahoo.com.br; lucianosrangel@yahoo.com.br; 1tstutz@iprj.uerj.br 
de danos estruturais e monitoramento de estruturas fundamentadas no domínio modal - domínio contendo os parâmetros modais da estrutura, quais sejam: frequências naturais, razões de amortecimento e formas modais - são constantes na literatura especializada, [1] e [13], com aplicações bem sucedidas nas engenharias mecânica, civil e aeroespacial [15]. O presente trabalho considera parâmetros modais da estrutura, especificamente, as frequências naturais não amortecidas.

Técnicas de identificação de danos estruturais e monitoramento de estruturas fundamentadas no ajuste de um MEF são constantes na literatura especializada [10]. No entanto, a obtenção de um problema geralmente mal posto, isto é, que apresenta não unicidade e instabilidade na solução, e o elevado custo computacional, inerente a essas técnicas, limitam ou até mesmo inviabilizam a sua aplicabilidade em estruturas que demandam um modelo de ordem elevada. Para contornar essas dificuldades pode-se utilizar o MSR em substituição a um MEF da estrutura [6], onde o MSR apresenta como vantagem a redução do custo computacional para a solução de problemas inversos de identificação de danos [2]. Segundo [14], o MSR tem como vantagens o baixo custo computacional e a aplicabilidade em problemas lineares e não-lineares, além da vantagem de relacionar os parâmetros de entrada para funções de saída de forma funcional, sendo assim as sensibilidades são facilmente identificáveis.

No presente trabalho, a identificação de danos estruturais considera o ajuste de um MSR da estrutura, objetivando-se a minimização de uma função de erro definida a partir das frequências naturais experimentais e das correspondentes frequências previstas pelo MSR. Estuda-se o problema de identificação de danos estruturais em uma viga de Euler-Bernoulli simplesmente apoiada considerando as frequências naturais na formulação do problema inverso.

O trabalho é organizado como se segue. Na Seção 2 é apresentado o modelo matemático utilizado para descrever o dano estrutural, assim como o problema de autovalor-autovetor, necessário para a obtenção das frequências naturais da estrutura. Na Seção 3, é descrita a base teórica do Modelo de Superfície de Resposta. A formulação do problema inverso de identificação de danos e o método estocástico de otimização ED são apresentados na Seção 4. A Seção 5 apresenta os resultados numéricos da analise do comportamento da viga em função da localização e da intensidade do dano e são apresentados, também, os resultados de identificação de danos obtidos a partir da consideração do Modelo de Superfície de Resposta e do método de otimização ED.

\section{FORMULAÇÃO DO PROBLEMA DIRETO}

Na estratégia de identificação de danos adotada, a integridade da estrutura é considerada como sendo continuamente descrita, no domínio do corpo, por um parâmetro estrutural denominado parâmetro nodal de coesão $(\beta)$ [12]. Este parâmetro está relacionado com a ligação entre os pontos materiais e pode ser interpretado como uma medida do estado de coesão local do material, onde $0 \leq \beta \leq 1$. Se $\beta=1$, considera-se que todas as ligações entre os pontos materiais foram preservadas, ou seja, não há defeito na estrutura. Se $\beta=0$, considera-se uma ruptura local, pois todas as ligações entre os pontos materiais foram desfeitas. 
Neste trabalho, considerou-se que o dano afeta apenas as propriedades elásticas da estrutura, hipótese comumente adotada na literatura. Deste modo, a matriz de rigidez do MEF da estrutura pode ser escrita como

$$
\mathbf{K}(\beta)=\int_{\Omega} \beta(x) E_{0} I_{0} \mathbf{H}^{T}(x) \mathbf{H}(x) d \Omega,
$$

onde $\mathbf{H}$ é o operador diferencial discretizado, $E_{0}$ e $I_{0}$ são, respectivamente, os valores nominais do módulo de elasticidade e do momento de inércia de área e $\beta$ representa o campo de coesão no domínio elástico $\Omega$ da estrutura. Deve-se enfatizar que a discretização do campo de coesão $\beta$ não depende da discretização do campo de deslocamentos, de forma que diferentes malhas podem ser adotadas.

Considerando um elemento de viga, de comprimento $l_{e}$, com dois graus de liberdade por nó e o campo de coesão, em seu interior, interpolado por funções lagrangeanas lineares a partir dos parâmetros nodais $\beta_{1}$ e $\beta_{2}$, tem-se a seguinte matriz de rigidez elementar

$$
\mathbf{K}^{e}=\frac{E_{0} I_{0}}{2 l_{e}}\left[\begin{array}{cccc}
\frac{12}{l_{e}^{2}}\left(\beta_{1}+\beta_{2}\right) & \frac{4}{l_{e}}\left(2 \beta_{1}+\beta_{2}\right) & -\frac{12}{l_{e}^{2}}\left(\beta_{1}+\beta_{2}\right) & \frac{4}{l_{e}}\left(\beta_{1}+2 \beta_{2}\right) \\
\frac{4}{l_{e}}\left(2 \beta_{1}+\beta_{2}\right) & 2\left(3 \beta_{1}+\beta_{2}\right) & -\frac{4}{l_{e}}\left(2 \beta_{1}+\beta_{2}\right) & 2\left(\beta_{1}+\beta_{2}\right) \\
-\frac{12}{l_{e}^{2}}\left(\beta_{1}+\beta_{2}\right) & -\frac{4}{l_{e}}\left(2 \beta_{1}+\beta_{2}\right) & \frac{12}{l_{e}^{2}}\left(\beta_{1}+\beta_{2}\right) & -\frac{4}{l_{e}}\left(\beta_{1}+2 \beta_{2}\right) \\
\frac{4}{l_{e}}\left(\beta_{1}+2 \beta_{2}\right) & 2\left(\beta_{1}+\beta_{2}\right) & -\frac{4}{l_{e}}\left(\beta_{1}+2 \beta_{2}\right) & 2\left(\beta_{1}+3 \beta_{2}\right)
\end{array}\right] .
$$

A partir da equação (2.1), tem-se que a rigidez à flexão ao longo da viga é dada por

$$
E(x) I(x)=\beta(x) E_{0} I_{0} .
$$

Portanto, o parâmetro de coesão representa qualquer alteração, provocada pela presença de danos estruturais, na rigidez à flexão da estrutura. Por simplicidade, considerando-se uma viga de seção transversal retangular e com módulo de elasticidade uniforme, o campo de coesão pode ser escrito como

$$
\beta(x)=\left(\frac{h(x)}{h_{0}}\right)^{3},
$$

onde $h_{0}$ e $h(x)$, indicam, respectivamente, a espessura nominal e a espessura da viga na posição $x$. O vetor de parâmetros nodais de coesão é definido como

$$
\boldsymbol{\beta}=\left[\begin{array}{llll}
\beta_{1} & \beta_{2} & \ldots & \beta_{n p}
\end{array}\right]^{T},
$$

onde $n p$ é o número total de parâmetros de coesão do modelo. Portanto, considerando-se as equações (2.2) e (2.3), nos nós defeituosos tem-se $h(x) / h_{0}<1$, e nos nós onde não há danos, 
tem-se $h(x) / h_{0}=1$. Sendo assim, a identificação de danos dar-se-à a partir da estimação do vetor de parâmetros nodais de coesão, uma vez que estes descrevem a integridade estrutural.

No presente trabalho, as frequências naturais não-amortecidas serão utilizadas no problema de identificação de danos estruturais, sendo estas, obtidas a partir do seguinte problema de autovalor-autovetor generalizado, escrito na forma matricial como

$$
\mathbf{K} \Phi=\mathbf{M} \Phi \Lambda,
$$

onde $\mathbf{M}$ é a matriz de massa e $\boldsymbol{\Phi}$ é a matriz modal da estrutura cuja $i$-ésima coluna corresponde à forma modal $\phi$ (i-ésimo autovetor) dada por

$$
\boldsymbol{\Phi}=\left[\begin{array}{llll}
\phi_{1} & \phi_{2} & \ldots & \phi_{n}
\end{array}\right]
$$

e $\boldsymbol{\Lambda}$ é uma matriz diagonal cujo $i$-ésimo componente (autovalor) é dado pelo valor quadrático da correspondente frequência natural não-amortecida do sistema, ou seja

$$
\boldsymbol{\Lambda}=\operatorname{diag}\left(\omega_{i}^{2}\right), \quad i=1, \ldots, n,
$$

sendo $\omega_{i}$ as frequências naturais não-amortecidas, onde as matrizes têm dimensão $n \times n$.

\section{MODELO DE SUPERFÍCIE DE RESPOSTA}

No MSR, relações explícitas são definidas entre parâmetros da estrutura - no caso especial de identificação de danos, os parâmetros nodais de coesão - e respostas de interesse. Desta forma, seja no domínio do tempo ou da frequência, para uma dada resposta escalar $y$, tem-se

$$
y=f\left(\beta_{1}, \beta_{2}, \ldots, \beta_{n p}\right)+\varepsilon
$$

onde $f\left(\beta_{1}, \beta_{2}, \ldots, \beta_{n p}\right)$ representa a relação entre a resposta e as variáveis independentes e $\varepsilon$ sendo o resíduo. Em geral, os fatores (parâmetros do modelo) são codificados como,

$$
x_{i}=\frac{\beta_{i}-\left(\beta_{\min }+\beta_{\max }\right) / 2}{\left(\beta_{\max }-\beta_{\min }\right) / 2}, \quad i=1,2, \ldots, n p,
$$

tal que $x_{i} \in[-1,1]$. Dessa forma, a equação (3.1) pode ser reescrita como

$$
y=f\left(x_{1}, x_{2}, \ldots, x_{n p}\right)+\varepsilon
$$

Na maioria dos casos, desconhece a relação entre a resposta e as variáveis independentes. Esta relação pode ser aproximada por polinômios de baixa ordem em algumas regiões relativamente pequenas do espaço definido pelas variáveis independentes, sendo então comumente utilizados modelos de primeira ou segunda ordem. Em geral, descreve-se o modelo de segunda ordem como

$$
y=b_{0}+\sum_{i=1}^{n p} b_{i} x_{i}+\sum_{i=1}^{n p} b_{i i} x_{i}^{2}+\sum_{i<j}^{n p} \sum_{j=2}^{n p} b_{i j} x_{i} x_{j}+\varepsilon,
$$

onde $b_{0}, b_{i}, b_{i i}$ e $b_{i j}$ são os coeficientes da função de resposta. 
Para a determinação dos coeficientes da função de resposta, deve-se ter um número $n_{d}$ de dados maior, ou no mímino igual, ao número de coeficientes. Considerando a equação (3.2) e um conjunto com $n_{d}$ dados, tem-se

$$
\mathbf{y}=\mathbf{X b}+\varepsilon,
$$

onde $\mathbf{y}$ é o vetor contendo os $n_{d}$ valores da resposta $y, \mathbf{X}$ é a denominada matriz de projeto, cujos componentes são obtidos dos parâmetros codificados $x_{i}$, b o vetor contendo os coeficientes da função de resposta e $\varepsilon$ o vetor contendo os resíduos do modelo.

Considerando o método dos mínimos quadrados, estima-se o vetor de coeficientes $\hat{b}$ por

$$
\hat{b}=\left(\mathbf{X}^{T} \mathbf{X}\right)^{-1} \mathbf{X}^{T} \mathbf{y}
$$

onde $T$, como usual, representa a operação de transposição de matriz. Portanto, a resposta $\hat{\boldsymbol{y}}$ prevista pelo modelo, pode ser representada como

$$
\hat{y}=\hat{b}_{0}+\sum_{i=1}^{n p} \hat{b}_{i} x_{i}+\sum_{i=1}^{n p} \hat{b}_{i i} x_{i}^{2}+\sum_{i<j}^{n p} \sum_{j=2}^{n p} \hat{b}_{i j} x_{i} x_{j} .
$$

Para cada resposta escalar considerada, determina-se uma superfície de resposta. Por simplicidade, será denominado MSR o conjunto envolvendo todas de superfícies de reposta em questão.

Deve-se notar que o aumento do número de parâmetros do modelo resulta em um aumento no número de coeficientes das superfícies e, principalmente, em um aumento significativo do número de possíveis combinações dos parâmetros. No entanto, a aplicação do modelo de superfície de resposta em estruturas mais complexas pode ser viabilizada, por exemplo, através da combinação de um modelo de dano constante por elemento (modelo mais simples que o adotado neste trabalho) e a definição de subestruturas. A utilização de um modelo de dano constante por elemento resulta em um menor número de possíveis combinações dos parâmetros que definem o dano. A utilização de subestruturas, por sua vez, também reduz consideravelmente o número de parâmetros considerado no problema inverso de identificação de danos. Por exemplo, como será apresentado posteriormente, no caso de uma viga simplesmente apoiada, danos de mesma magnitude e em posições simétricas resultam nas mesmas variações nas frequências naturais e, portanto, uma estratégia de identificação de danos baseada apenas nas frequências naturais é, em princípio, incapaz de diferenciar esses cenários. Sendo assim, elementos simétricos em uma viga simplesmente apoiada podem ser agrupados de modo a formar uma subestrutura. Uma vez formadas as subestruturas, apenas um elemento de cada subestrutura pode ser considerado no processo de identificação de danos, reduzindo, portanto, de forma significativa o número de parâmetros considerado.

Para a determinação dos coeficientes do MSR, é usual a utilização de técnicas de Projeto de Experimentos. Existem dois tipos principais de projeto: Projeto Composto Central (PCC) e Box-Behnken [5]. Um modelo de primeira ou de segunda ordem, pode ser construído de forma eficiente com o PCC, sendo assim, utiliza-se este no presente trabalho. 


\subsection{Projeto Composto Central}

O PCC compõe um Projeto de Experimentos utilizado para ajustar um modelo de superfície de resposta de primeira ou de segunda ordem [8]. Constitui-se de três partes um PCC para $n p$ fatores, devidamente codificados como $x_{1}, x_{2}, \ldots, x_{n p}$, como se segue: uma parte fatorial, contendo um total $2^{n p}$ pontos de coordenadas $x_{i}=-1$ e $x_{i}=+1$, para $i=1,2, \ldots, n p$; uma parte axial, formada por $2 n p$ pontos com todas as coordenadas nulas, exceto um, que possui um valor $\alpha$ (ou $-\alpha$ ); um total de $m$ ensaios realizados no ponto central, onde, $x_{1}=\ldots=x_{n p}=0$.

O total de combinações previstas no PCC, considerando-se as combinações anteriormente citadas, é dado por

$$
N_{c}=2^{n p}+2 n p+m
$$

Define-se $\alpha$ como sendo a distância do ponto central aos pontos axiais, implicando-se em um PCC rotacional (ou não). Quando todos os pontos estiverem à mesma distância do ponto central, estes terão o desvio padrão da resposta prevista constante, classificando-se assim, o PCC em rotacional.

Classifica-se um PCC em três tipos: Circunscrito (CCC), Inscrito (CCI) e Face Centrada (CCF). No CCC os pontos axiais estão a uma distância $\alpha$ do ponto central, sendo assim, fornecem novos extremos para os níveis alto e baixo dos fatores, logo, os níveis $[-1,+1]$ dos fatores são extrapolados. Além dos dois níveis axiais, cada fator requer mais três níveis, dois fatoriais $[-1,+1]$ e um do ponto central, totalizando cinco níveis. Este tipo de PCC para dois fatores pode ser visualizado na Figura 1.

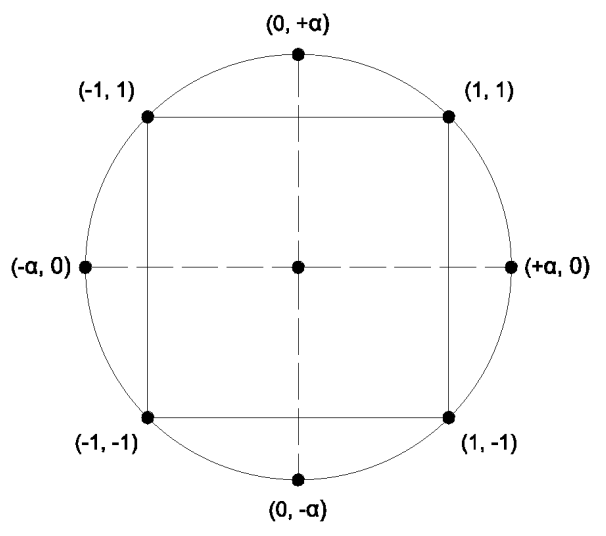

Figura 1: PCC Circunscrito.

No CCI os níveis dos fatores $[-1,+1]$ são usados como pontos axiais e os pontos fatoriais são criados dentro destes limites (níveis dos fatores originalmente especificados), este projeto também contém o ponto central, totalizando cinco níveis para cada fator. O PCC inscrito para dois fatores pode ser visualizado na Figura 2. 


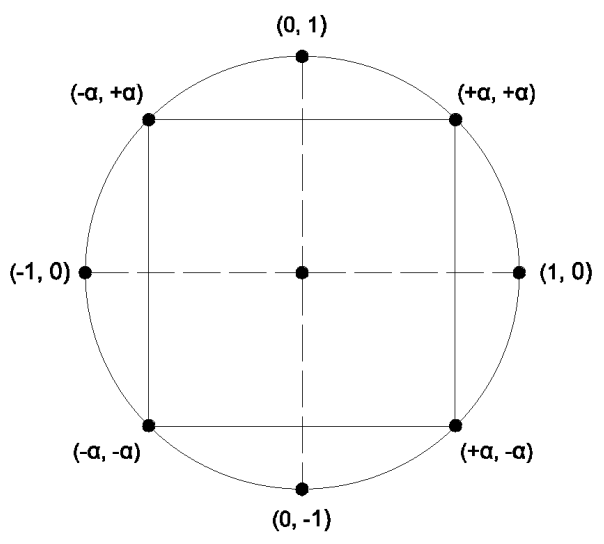

Figura 2: PCC Inscrito.

Por fim, no CCF os pontos axiais estão no centro de cada face do espaço fatorial, neste caso, $\alpha= \pm 1$. Assim sendo, neste projeto, utiliza-se pontos no domínio original definido pelos níveis dos fatores. Neste caso, para cada fator necessita-se de apenas três níveis. O CCF para dois fatores pode ser visualizado na Figura 3.

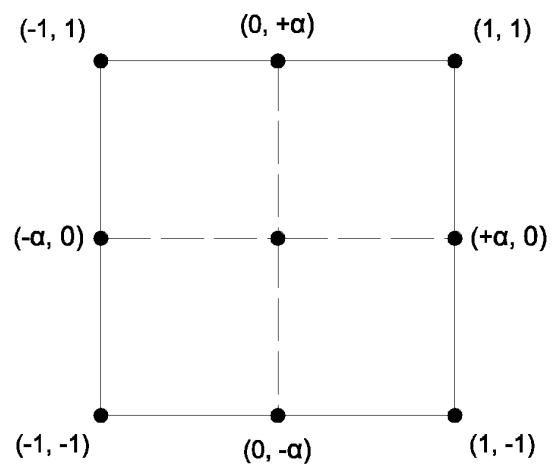

Figura 3: PCC Face Centrada.

\section{FORMULAÇÃO DO PROBLEMA INVERSO}

No presente trabalho, o problema de identificação de danos é definido como um problema de otimização, onde o objetivo é minimizar um funcional definido a partir da diferença entre as frequências naturais experimentais e as correspondentes frequências previstas por um MSR da estrutura.

Para a formulação do problema de identificação de danos baseado nas frequências naturais da estrutura, define-se o vetor de resposta generalizada como,

$$
\mathbf{v}=\left[\begin{array}{llll}
\omega_{n_{1}} & \omega_{n_{2}} & \ldots & \omega_{n_{E}}
\end{array}\right],
$$


onde $\omega_{i}, i=n_{1}, \ldots, n_{E}$, representa a $i$-ésima frequência natural da estrutura e $n_{E}$ é o número de frequências naturais consideradas no processo de identificação. Na definição do problema de identificação de danos, tem-se um funcional definido a partir da diferença entre a resposta experimental e a reposta prevista pelo MSR,

$$
\min _{\boldsymbol{\beta}} \mathcal{F}
$$

onde

$$
\mathcal{F}=\left(\mathbf{v}_{E}-\hat{\mathbf{v}}(\boldsymbol{\beta})\right)^{T} \mathbf{W}\left(\mathbf{v}_{E}-\hat{\mathbf{v}}(\boldsymbol{\beta})\right),
$$

e representa a norma quadrática ponderada da diferença entre o vetor contendo as frequências naturais obtidas experimentalmente $\left(\mathbf{v}_{E}\right)$ e o vetor contendo as frequências naturais previstas pelo MSR $(\hat{\mathbf{v}}(\boldsymbol{\beta}))$, ou seja, para cada frequência natural que compõe o vetor $\mathbf{v}$, determina-se uma superfície de resposta, e $\mathbf{W}$ é uma matriz de ponderação, dada por

$$
\mathbf{W}=\left[\begin{array}{cccc}
\frac{1}{\omega_{n 1}} & 0 & \ldots & 0 \\
0 & \frac{1}{\omega_{n 2}} & \cdots & 0 \\
\vdots & & \ddots & 0 \\
0 & \ldots & 0 & \frac{1}{\omega_{n_{E}}}
\end{array}\right]
$$

O problema inverso em questão é definido considerando um MSR da estrutura, onde o MSR representa de forma explícita as relações entre os parâmetros nodais de coesão e as respostas de interesse, sendo estas relações dadas por polinômios, o que, por sua vez, reduz de forma significativa o esforço computacional na resolução do problema direto [9]. No presente trabalho, para a resolução do problema inverso, utilizou-se o algoritmo ED.

\subsection{Evolução Diferencial}

Em sua forma clássica, o método de otimização estocástico ED, proposto por [11], baseia-se na geração de novos indivíduos, denominados vetores doadores, pela adição da diferença vetorial ponderada entre dois indivíduos aleatórios da população a um terceiro indivíduo.

Seja uma população inicial, comumente gerada por uma distribuição de probabilidade uniforme, constituída por $N_{p}$ indivíduos (com $n p$ componentes representando cada variável do projeto), chamados vetores, percorrendo todo o espaço de busca. Portanto, a população segue uma evolução natural, todavia, a quantidade de indivíduos permanece fixa durante todo o processo de minimização.

Vale destacar, que cada indivíduo da população inicial, representa um possível candidato à solução do problema de otimização, como pode ser observado pela equação (4.1),

$$
\boldsymbol{\beta}_{i}=\left[\begin{array}{lllll}
\beta_{1} & \beta_{2} & \ldots & \beta_{n p-1} & \beta_{n p}
\end{array}\right]^{T} .
$$

O ED possui como ideia básica a geração de novos indivíduos, denotados vetores modificados ou doadores, pela adição da diferença vetorial ponderada entre dois indivíduos aleatórios da 
população a um terceiro indivíduo (na sua forma trivial). Comumente, chama-se esta operação de mutação.

As componentes deste novo indivíduo doador são permutadas com as componentes de um indivíduo selecionado aleatoriamente (denotado vetor alvo ou vetor a ser substituído), para transformar no vetor tentativa (vetor experimental). Frequentemente cita-se o processo de permutar as componentes do indivíduo doador com as componentes do vetor alvo, como cruzamento no grupo dos algoritmos evolutivos.

Se o vetor tentativa produzir um valor na função objetivo menor do que o valor do vetor alvo, então o vetor tentativa, passa a ser o vetor alvo da geração seguinte. Esta última operação é chamada de seleção. Finaliza-se o procedimento através da algum critério de parada. Observa-se claramente que a população passa por três estágios antes de produzir a próxima geração, que são: mutação, cruzamento e seleção.

Mutação: para a aquisição do vetor doador $\mathbf{V}^{(q+1)}$, considere os vetores $\mathbf{A}_{\delta}^{(q)}, \mathbf{A}_{\rho}^{(q)}$ e $\mathbf{A}_{\gamma}^{(q)}$ distintos entre si e escolhidos aleatoriamente em uma população com $N_{p}$ indivíduos. Os índices aleatórios $\delta, \rho, \gamma$ pertencente ao conjunto $\left[1, N_{p}\right]$, são inteiros e distintos entre si. Usando-se o par de vetores $\left(\mathbf{A}_{\rho}, \mathbf{A}_{\gamma}\right)$ da q-ésima geração, define-se o vetor diferença $\left(\mathbf{A}_{\rho}-\mathbf{A}_{\gamma}\right)$. Multiplica-se esta diferença por $F_{p}$ (fator de perturbação - número real pertencente ao intervalo [0,2] e controla a amplitude do vetor diferença), sendo denotada diferença vetorial ponderada e a utiliza-se para perturbar o terceiro vetor $\mathbf{A}_{\delta}$.

Expressa-se a mutação como

$$
\mathbf{V}^{(q+1)}=\mathbf{A}_{\delta}^{(q)}+F_{p}\left(\mathbf{A}_{\rho}^{(q)}-\mathbf{A}_{\gamma}^{(q)}\right) .
$$

Cruzamento: supondo-se que para cada vetor alvo $\mathbf{A}_{s}^{(q)}$, com $s$ pertencente ao intervalo $\left[1, N_{p}\right]$ e distintos dos índices $\delta, \rho$ e $\gamma$, gerou-se um vetor doador. Insere-se o cruzamento para ampliar a diversidade dos indivíduos que sofreram a mutação. Escolhe-se pela equação (4.2), as componentes do vetor tentativa $\mathbf{U}^{(q+1)}$, oriundos do vetor doador e do vetor alvo.

$$
\mathbf{U}(i)^{(q+1)}=\left\{\begin{array}{l}
\mathbf{V}(i)^{(q+1)}, \text { se } \operatorname{rand}_{i} \leq P_{c} \text { ou } i=R, \\
\mathbf{A}_{s}(i)^{(q)}, \text { se } \operatorname{rand}_{i}>P_{c} \text { e } i \neq R, i=1, \ldots, n .
\end{array}\right.
$$

onde, $\mathbf{V}(i)^{(q+1)}$ é a $(q+1)$-ésima componente do vetor doador $\mathbf{V}^{(q+1)}, \mathbf{A}_{s}(i)$ a do vetor alvo $\mathbf{A}_{s}^{(q)}, \operatorname{rand}_{i}$ um número gerado aleatoriamente no intervalo $[0,1]$ e $R$ um índice escolhido aleatóriamente, $R \in 1,2, \ldots, n p$. Define-se $P_{c}$, como a probabilidade de cruzamento (fornecido pelo usuário e compreendido no intervalo entre $[0,1]$ ), simbolizando-se a chance do vetor tentativa herdar os valores das variáveis do vetor doador. Denomina-se este tipo de cruzamento, apresentado por [11], como cruzamento binomial.

Seleção: processo de procriar filhos melhores. Avalia-se o custo do vetor tentativa $\mathbf{U}^{(q+1)}$ e compara-se com o custo do vetor alvo $\mathbf{A}_{s}^{(q)}$. Se o custo do vetor tentativa for menor que o custo do vetor alvo, o vetor alvo passa a ser o vetor tentativa da próxima geração. Caso contrário, o vetor alvo da próxima geração é o mesmo vetor alvo da geração atual. 
Resume-se a seleção por:

$$
\left\{\begin{array}{l}
\operatorname{Se} f\left(\mathbf{U}^{(q+1)}\right) \leq f\left(\mathbf{A}_{s}^{(q)}\right) \text { então } \mathbf{A}_{s}^{(q+1)}=\mathbf{U}^{(q+1)} \\
\operatorname{Se} f\left(\mathbf{U}^{(q+1)}\right)>f\left(\mathbf{A}_{s}^{(q)}\right) \text { então } \mathbf{A}_{s}^{(q+1)}=\mathbf{A}_{s}^{(q)}
\end{array}\right.
$$

Após os estágios (mutação, cruzamento e seleção), passa-se então para a próxima geração e todos os procedimentos anteriores são refeitos. Deve-se adotar critério(s) de parada(s), sendo que o número máximo de gerações deve ser estabelecido.

Habitualmente, a evolução do algoritmo de ED depende, sobretudo, do tamanho da população $N_{p}$, da região de busca, da taxa de cruzamento e também do fator de perturbação $F_{p}$.

\section{RESULTADOS NUMÉRICOS}

Nos resultados que se seguem, considerou-se uma viga de aço simplesmente apoiada com os parâmetros geométricos e materiais apresentados na Tabela 1.

Tabela 1: Propriedades nominais da viga.

\begin{tabular}{|c|c|}
\hline Comprimento & $1,46 \mathrm{~m}$ \\
\hline Espessura & $7,9375 \times 10^{-3} \mathrm{~m}$ \\
\hline Largura & $7,62 \times 10^{-2} \mathrm{~m}$ \\
\hline Momento de Inércia de Área & $3,1756 \times 10^{-9} \mathrm{~m}^{4}$ \\
\hline Módulo de Elasticidade & $2,07 \times 10^{11} \mathrm{~Pa}$ \\
\hline Massa específica & $7,85 \times 10^{3} \mathrm{~kg} / \mathrm{m}^{3}$ \\
\hline
\end{tabular}

A viga em questão foi discretizada pelo MEF em 24 elementos bidimensionais do tipo EulerBernoulli, onde cada elemento apresenta dois nós e cada nó possui dois graus de liberdade (GDL) - um de rotação e outro de translação - e um parâmetro de coesão. Portanto, a estrutura possui 25 parâmetros nodais de coesão e, devido às condições de contorno, 48 GDL.

Nos casos considerados, utiliza-se a mesma malha de elementos para aproximar o campo de deslocamentos e também o campo de coesão (dano). O MEF é utilizado com os valores nodais do parâmetro de coesão prescrito de acordo com os cenários de dano considerados, para gerar as frequências naturais da estrutura danificada, representado assim, os dados experimentais sintéticos utilizados no processo de identificação de danos.

A Tabela 2 apresenta as quatro primeiras frequências naturais não amortecidas e os correspondentes erros relativos, com respeito às frequências naturais teóricas, para a viga não danificada.

Pela Tabela 2, observa-se que os erros relativos das quatro primeiras frequências naturais, apresentam magnitude pequena, indicando assim, acurácia no modelo adotado. 
Tabela 2: Frequências naturais da viga simplesmente apoiada sem dano.

\begin{tabular}{|c|c|c|c|}
\hline $\begin{array}{c}\text { Modo de } \\
\text { Vibração }\end{array}$ & $\begin{array}{c}\text { Frequência Natural } \\
\text { Teórica (Hz) }\end{array}$ & $\begin{array}{c}\text { Frequência } \\
\text { Natural (Hz) }\end{array}$ & $\begin{array}{c}\text { Erro } \\
\text { Relativo (\%) }\end{array}$ \\
\hline 1 & 8,67077 & 8,67077 & 0,00002 \\
\hline 2 & 34,68308 & 34,68319 & 0,00032 \\
\hline 3 & 78,03692 & 78,03821 & 0,00164 \\
\hline 4 & 138,73231 & 138,73949 & 0,00517 \\
\hline
\end{tabular}

No presente trabalho, objetivando simular de forma mais realística os dados experimentais, considerou-se a presença de ruído aditivo, de média nula, nas frequências naturais experimentais sintéticas como se segue

$$
\omega_{i, \exp }=\omega_{i, \exp }^{0}\left(1+\frac{p}{100} \operatorname{rand}(-1,1)\right),
$$

sendo $\omega_{i, \exp }$ e $\omega_{i, \exp }^{0}$, as frequências naturais experimentais sintéticas contaminadas com ruído e as frequências naturais sem ruído, respectivamente, $p$ representa o nível de ruído acrescentado e rand $(-1,1)$ representa uma função geradora de números aleatórios, de média nula e distribuição uniforme, no intervalo de $(-1,1)$.

Uma análise do comportamento da viga é realizada, em relação às suas frequências naturais, em função da posição e da intensidade do dano. Considera-se cada posição nodal da malha de defeitos, uma por vez, para danos de intensidade leve $\left(h(x) / h_{0}=0,9\right)$, média $\left(h(x) / h_{0}=\right.$ $0,75)$ e severa $\left(h(x) / h_{0}=0,5\right)$. Para cada modo de vibração considerado, é calculada a razão entre as frequências naturais da estrutura com e sem dano,

$$
\omega_{R_{i}}=\frac{\omega_{d_{i}}}{\omega_{i}}
$$

sendo $\omega_{d_{i}}$ e $\omega_{i}$, a frequência natural da estrutura com e sem dano para o i-ésimo modo de vibração, respectivamente. A Figura 4, apresenta a influência da intensidade e localização do dano nas duas primeiras frequências naturais da estrutura.

Na Figura 4, é possível observar que as frequências naturais apresentam pouca sensibilidade aos danos nas regiões próximas ao extremo da viga, sendo provável uma maior dificuldade na identificação de danos nessas regiões. Ademais, observa-se que danos de mesma magnitude e em posições simétricas resultam nas mesmas variações nas frequências naturais e, portanto, uma estratégia de identificação de danos baseada apenas nas frequências naturais é, em princípio, incapaz de diferenciar esses cenários. Sendo assim, elementos simétricos da viga são agrupados de modo a formar subestruturas e apenas um elemento de cada subestrutura é considerado no processo de identificação de danos, reduzindo, portanto, de forma significativa o número de parâmetros de coesão considerado no problema inverso. Como apenas as frequências naturais da viga são consideradas no problema inverso de identificação de danos, nos resultados aqui apresentados tem-se a intensidade do dano e as subestruturas supostamente danificadas, não sendo possível diferenciar, nessas subestruturas, quais elementos realmente apresentam danos. 

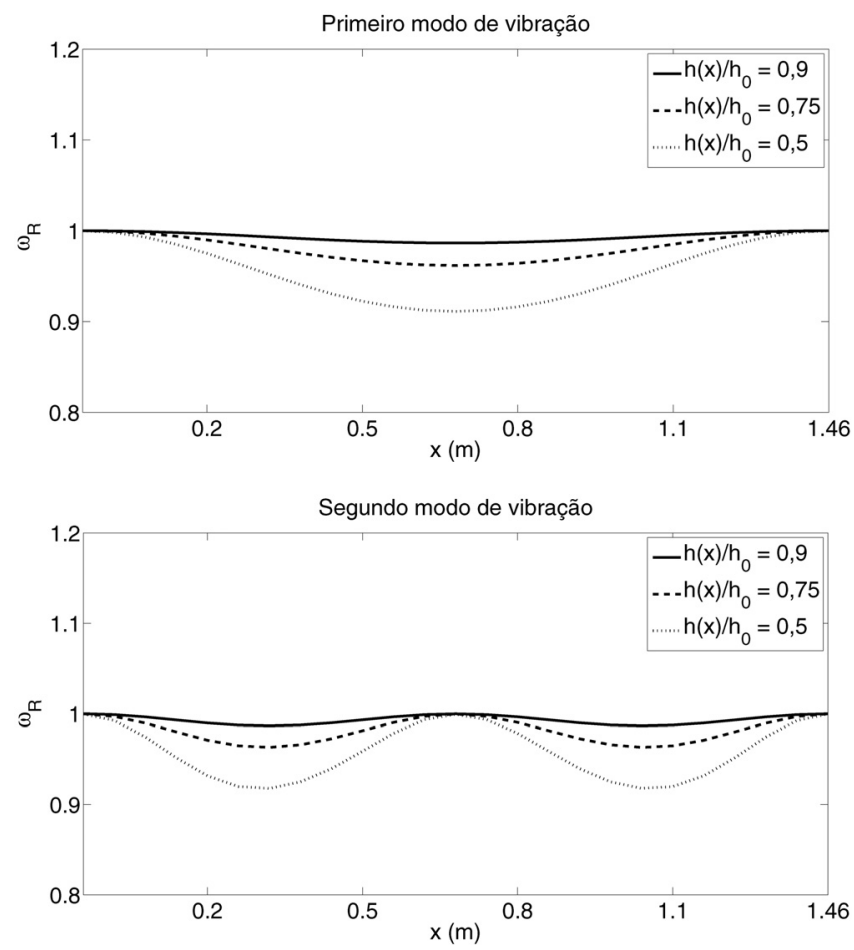

Figura 4: Influência da posição e intensidade do dano nas frequências naturais da estrutura.

A viga em questão foi discretizada em 24 elementos, resultando em 25 parâmetros nodais de coesão. Para a resolução do problema de identificação de danos, foram definidas 13 subestruturas, conforme ilustrado na Figura 5.

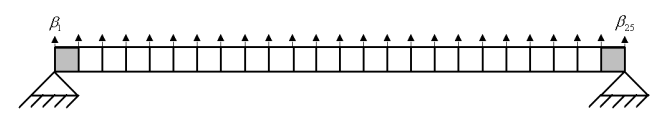

(a) Subestrutura 1

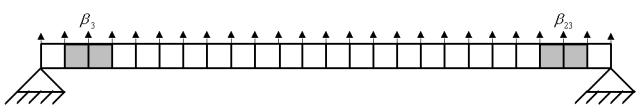

(c) Subestrutura 3

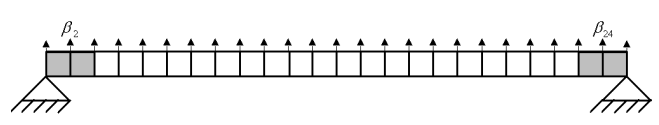

(b) Subestrutura 2

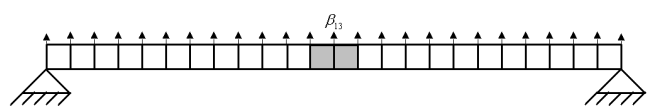

(d) Subestrutura 13

Figura 5: Divisão das subestruturas presentes na viga simplesmente apoiada.

Para a obtenção do MSR utilizou-se o projeto CCC. Este projeto necessita da definição dos pontos fatoriais $\left(\beta_{-1}, \beta_{1}\right)$, pontos axiais $\left(\beta_{-\alpha}, \beta_{\alpha}\right)$ e ponto central $\left(\beta_{0}\right)$, vide Figura 1 . Os valores considerados foram: $\beta_{-\alpha}=0,182, \beta_{-1}=0,512, \beta_{0}=0,729, \beta_{1}=1$ e $\beta_{\alpha}=1,331$. Se todos os parâmetros do coesão fossem considerados na realização de um projeto $\mathrm{CCC}$, de acordo 
com a equação (3.3), seria possível um total de 33554483 combinações. Considerando-se as 13 subestruturas, o número de combinações do planejamento diminui consideravelmente para 8219 combinações.

A princípio foram considerados três tipos de MSR: linear (LI), quadrático sem interação entre os parâmetros $(\mathrm{QP})$ e quadrático com interação entre os parâmetros (QI). Depois de algumas análises realizadas, constatou-se que a superfície QI reproduz o melhor ajuste do modelo. $\mathrm{Na}$ identificação de danos utiliza-se o MSR das quatro primeiras frequências naturais, sendo estas superfícies do tipo QI e geradas a partir de um projeto D-ótimo com 200 combinações, onde estas combinações são escolhidas entre as 8219 combinações possíveis do CCC para as 13 subestruturas consideradas.

Considerou-se, que tanto na obtenção do modelo da estrutura utilizando-se o MSR quanto na obtenção dos dados sintéticos na identificação de danos, que apenas as frequências contidas na faixa de 0 a $200 \mathrm{~Hz}$ foram medidas, ou seja, foram consideradas as quatro primeiras frequências naturais. A Tabela 3, apresenta os cenários de danos que serão considerados neste trabalho.

Tabela 3: Cenários de danos - Viga simplesmente apoiada.

\begin{tabular}{|c|c|c|c|c|}
\hline Caso & Subestrutura & Posição $(\mathrm{m})$ & $h(x) / h_{0}$ & Nível de ruído $(\%)$ \\
\hline 1 & 10 & 0,5475 & 0,8 & 1 \\
\hline 2 & $10 ; 4$ & 0,$5475 ; 1,2775$ & 0,9 & 0 \\
\hline
\end{tabular}

No Caso 1 considerou-se apenas uma região danificada, definida por $h(x) / h_{0}=0,8$ (ou seja $\beta(x)=0,512$ ) em $x=0,5475 \mathrm{~m}$, e frequências naturais corrompidas com $1 \%$ de ruído. No Caso 2, realiza-se a imposição do dano através da redução de $10 \%$ na altura relativa da seção transversal nas duas posições danificadas, ou seja, $h(x) / h_{0}=0,9(\beta(x)=0,729)$ para $x=0,5475 \mathrm{~m}$ e $x=1,2775 \mathrm{~m}$, e não se considera ruído.

Devido à aleatoriedade dos métodos estocásticos, foram realizadas 10 simulações com o método ED e o resultado final apresentado foi obtido da média aritmética envolvendo os resultados parciais. Para a parametrização do método, foram utilizados os seguintes valores: tamanho da população 120 , fator de pertubação 0,5 , probabilidade de cruzamento 0,9 e número de gerações $q=1000$. O critério de parada adotado foi através do número máximo de gerações ou a tolerância do funcional de $10^{-15}$. Para validação do resultado, calculam-se os erros relativos das quatro primeiras frequências naturais, antes e depois da identificação de danos.

Na Figura 6, é apresentada a média das dez simulações utilizando o método ED e os erros relativos, para o Caso 1.

Pela Figura 6, verifica-se que tanto a localização quanto a intensidade do dano foram apresentados de forma satisfatória, logo, conclui-se que, o campo de dano estimado, recupera de forma correta o campo de dano exato na subestrutura danificada, mesmo considerando ruído aditivo. O resultado obtido é, de certa forma, validado pela diminuição do erro relativo das frequências naturais após a identificação. 

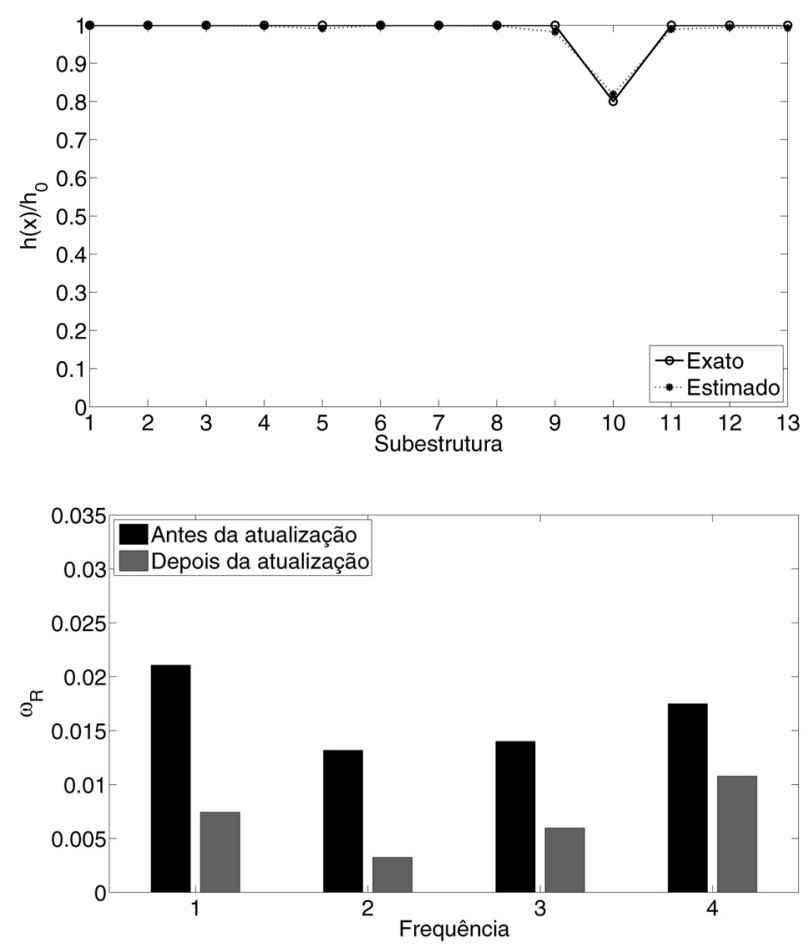

Figura 6: Identificação de danos para o Caso 1.

Na Figura 7, verifica-se a estratégia foi capaz de identificar tanto as subestruturas danificadas quanto a intensidade dos danos para o cenário do Caso 2.

Pode-se observar que a abordagem proposta foi capaz de identificar com acurácia as subestruturas danificadas e a intensidade dos danos. Com relação ao custo computacional, avaliou-se o tempo gasto no processo de identificação de danos tanto utilizando o MSR quanto MEF. Foi possível observar que em ambos casos estudados, o processo de identificação de danos na viga utilizando o MEF gastou em média 480 s, enquanto o MSR gastou em média $20 \mathrm{~s}$. A partir deste resultado, verifica-se que o MSR reduz consideravelmente o custo computacional na formulação de problemas de identificação de danos, conforme valida a literatura especializada [3].

\section{CONCLUSÕES}

A utilização das frequências naturais em um problema de identificação de danos estruturais mostrou-se bastante adequada, visto que as frequências naturais podem ser facilmente obtidas e que são pouco afetadas por erros de medição. No caso em questão, a viga foi considerada como sendo formada por subestruturas, diminuindo assim, significativamente o número de parâmetros nodais de coesão a serem considerados na geração das superfícies de resposta.

A partir dos resultados, pode-se concluir que a estratégia adotada é capaz de localizar e quantificar as subestruturas danificadas com elevada acurácia. Estes podem ser considerados como 

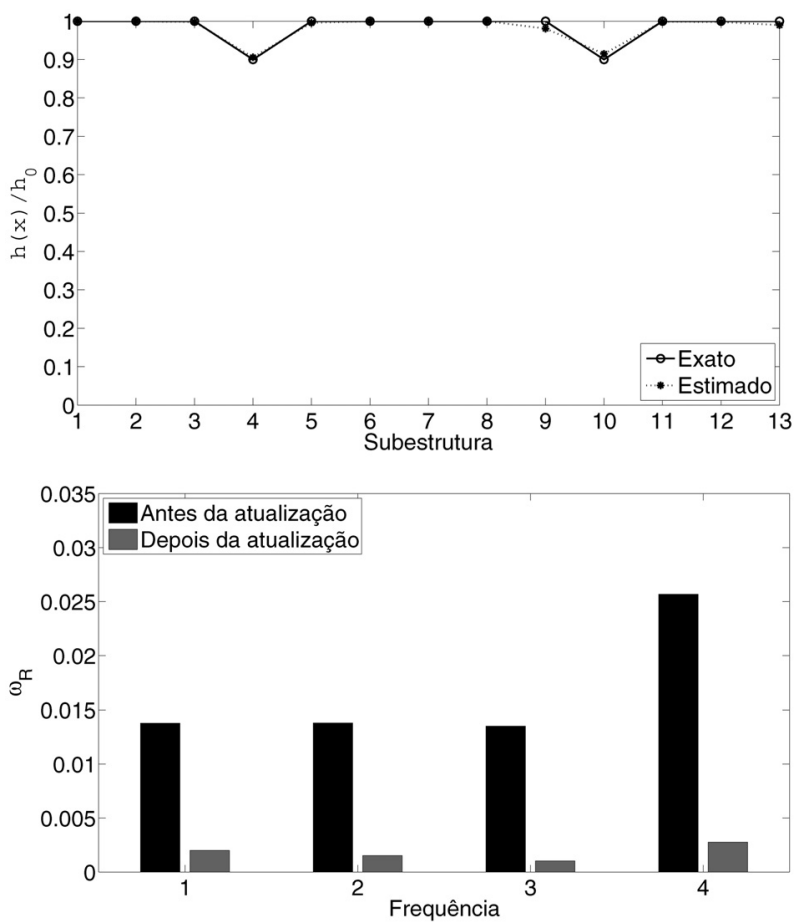

Figura 7: Identificação de danos para o Caso 2.

informação a priori em outras abordagens de identificação de danos, como aquelas baseadas em respostas dinâmicas temporais da estrutura. Ao se considerar as regiões potencialmente danificadas, apenas os parâmetros de coesão contidos nessas regiões e em uma vizinhança arbitrária podem ser estimados no processo de identificação de danos, o que pode reduzir de forma significativa o custo computacional para a resolução do problema inverso. Como sugestões para trabalhos futuros temos a aplicação do MSR em estruturas do tipo viga, com outras condições de contorno, e estruturas do tipo placa e construção de MSR baseados em respostas temporais da estrutura.

\begin{abstract}
The present work considers the structural damage identification problem built on the natural frequencies of vibration. In the formulation of the damage identification problem, a Response Surface Model (RSM) was used in place of a Finite Element Model (FEM) of the structure. The damage identification in a simply supported Euler-Bernoulli beam is studied, wherein the inverse problem is defined as a minimization one, whose goal is to minimize an objective function defined from the experimental natural frequencies and the corresponding natural frequencies prescribed by a RSM of the structure. The use of the Differential Evolution (DE) method in the inverse problem of damage identification is considered. Considering the numerical results obtained, the strategy adopted proved to be able to locate and quantify the damage with high accuracy, showing the capability of the proposed damage identification strategy.
\end{abstract}


Keywords: identification of structural damage, natural frequencies, response surface model.

\section{REFERÊNCIAS}

[1] A. Alvandi \& C. Cremona. Assessment of vibration-based damage identification techniques. Journal of Sound and Vibration, 202 (2006), 179-202.

[2] S.E. Fang \& R. Perera. A response surface methodology based damage identification technique. $J$. Iop Poblishing, 18 (2009), 14.

[3] S.E. Fang, R. Wei-Xin \& R. Perera. A stochastic model updating method for parameter variability quantification based on response surface models and Monte Carlo simulation. Mechanical Systems and Signal Processing, 33 (2012), 83-96.

[4] Q.T. Guo \& L.M. Zhang. Finite element model updating based on response surface methodology Proc. 22nd Int. Modal Analysis Conf. (Dearborn, MI), (2004).

[5] P.S. Lopes. Modelagem de problema inverso de detecção de danos por técnicas de identificação de parâmetros e de otimização. Tese de Doutorado em Engenharia Mecânica, Instituto de Engenharia Mecânica, Universidade Federal de Itajubá, (2010), pp. 135.

[6] C.C. Lin \& Y.J. Lee. Regression of the response surface of laminated composite structures. Composite Structures, 62 (2003), 91-105.

[7] L. Meirovitch. Elements of vibration analysis. New York: McGraw-Hill, (1986).

[8] D.C. Montgomery. Diseño y análisis de experimentos. Universidad Estatal de Arizona. Limusa Wiley, 2 ed., (1991), pp. 692.

[9] I.C.S.S. Rangel. Identificação de danos estruturais a partir do modelo de superfície de resposta. Dissertação de Mestrado em Modelagem Computacional, Instituto Politécnico, Universidade do Estado do Rio de Janeiro, Nova Friburgo, (2014), pp. 109.

[10] L.S. Rangel, I.C.S.S. Rangel \& L.T. Stutz. Identificação de danos estruturais utilizando dados no domínio do tempo e critério D-ótimo. Tendências em Matemática Aplicada e Computacional, 16 (2015), 209-218.

[11] R. Storn \& K. Price. Differential Evolution - A simple and efficient adaptive scheme for global optimization over continuous spaces. Journal of Global Optimization, 11 (1997), 341-359, Berkekey.

[12] L.T. Stutz, D.A. Castello \& F.A. Rochinha. A flexibility-based continuum damage identification approach. Journal of Sound and Vibration, 279 (2005), 641-667.

[13] L.T. Stutz, R.A. Tenenbaum \& R.A.P. Correa. The Differential Evolution method applied to continuum damage identification via flexibility matrix. Journal of Sound and Vibration, 345 (2015), 86-102.

[14] A.C. Rutherforda, D.J. Inmanb, G. Parka \& F.M. Hemeza. Use of response surface metamodels for identification of stiffness and damping coefficients in a simple dynamic system. Shock and Vibration, 12 (2005), 317-331.

[15] A. Tomaszewska. Influence of statistical errors on damage detection based on structural flexibility and mode shape curvature. Computers and Structures, 88 (2010), 154-164. 